\title{
Five-year real world outcomes of GeoForm ring implantation in patients with ischemic mitral regurgitation
}

\author{
Tomasz A. Timek, MD, ${ }^{\mathrm{a}}$ Robert L. Hooker, MD, ${ }^{\mathrm{a}}$ Robin Collingwood, MD, ${ }^{\mathrm{b}}$ Alan T. Davis, PhD, \\ Craig T. Alguire, MD, ${ }^{a}$ Charles L. Willekes, MD, ${ }^{a}$ Edward T. Murphy, MD, ${ }^{a}$ John C. Heiser, MD, ${ }^{a}$ and \\ Lawrence H. Patzelt, $\mathrm{MD}^{\mathrm{a}}$
}

\begin{abstract}
Background: Reductive ring annuloplasty represents the current standard surgical therapy for ischemic mitral regurgitation (IMR); however, the clinical results have been suboptimal. Etiology-specific prostheses such as the GeoForm annuloplasty ring have been designed to better address the annular and subvalvular perturbations associated with IMR. However, clinical experience is limited, and mid-term results are lacking.

Methods: We reviewed the clinical outcomes of 86 patients who had undergone implantation of a GeoForm ring at our center from 2005 to 2011. Perioperative mortality and clinical parameters were derived from The Society of Thoracic Surgeons database. Follow-up survival was assessed using the Social Security Death Index. Surviving patients were interviewed by telephone for valve-specific follow-up data and to complete the Medical Outcomes Study, short-form, 36-item, quality-of-life questionnaire.

Results: The mean grade of IMR preoperatively was $3.1 \pm 0.8$ (range, 1-4+), $0.2 \pm 0.4$ in the immediate postoperative period, and $0.7 \pm 0.7$ at the last mean follow-up point of 41 months; only 2 patients developed $\geq 2+$ IMR during the follow-up period, for a 5-year freedom from recurrent $2+$ IMR of $86 \%$. The mean left ventricular end-diastolic and end-systolic diameters decreased from before to after surgery from $6.0 \pm 0.0$ $\mathrm{cm}$ to $5.3 \pm 09 \mathrm{~cm}$ and $5.0 \pm 0.9 \mathrm{~cm}$ to $4.3 \pm 1.1 \mathrm{~cm}$, respectively $(P<.001)$. Perioperative mortality was $5.8 \%$ (5 of 86), and 1-, 3-, and 5-year survival was $87 \%, 81 \%$, and $75 \%$, respectively. At the last follow-up point, $80 \%$ of patients were in New York Heart Association class I and II, and their quality of life was equal to, or better than, age-matched controls from the general population.
\end{abstract}

Conclusions: Implantation of the GeoForm ring offers very good control of IMR, with low rate of recurrent IMR at mid-term follow-up. The use of this prosthesis was associated with good perioperative mortality, mid-term survival, and quality of life. (J Thorac Cardiovasc Surg 2014;148:1951-6)

Ischemic mitral regurgitation (IMR) continues to be a challenging clinical problem for surgeons and cardiologists alike. The etiology of IMR has yet to be clearly elucidated, although recent clinical ${ }^{1}$ and experimental ${ }^{2}$ data have significantly contributed to our understanding of the annular and subvalvular perturbations that contribute to its genesis. The current standard clinical repair of IMR has been centered around annular reduction using a complete undersize annuloplasty ring. However, the need for subvalvular intervention to treat the "ventricular" disease of IMR has

\footnotetext{
From the Department of Cardiothoracic Surgery, ${ }^{a}$ Meijer Heart and Vascular Institute at Spectrum Health, Grand Rapids, Mich; Michigan State University College of Human Medicine, ${ }^{\text {b }}$ Grand Rapids, Mich; and Grand Rapids Education Partners, ${ }^{c}$ Grand Rapids, Mich.

Disclosures: Authors have nothing to disclose with regard to commercial support.

Presented in part at The American Association for Thoracic Surgery Mitral Conclave,

May 2013, New York, NY.

Received for publication May 14, 2013; revisions received Feb 5, 2014; accepted for publication Feb 14, 2014; available ahead of print March 28, 2014

Address for reprints: Tomasz A. Timek, MD, Department of Cardiothoracic Surgery, Meijer Heart and Vascular Institute at Spectrum Health, 100 Michigan Ave, NE, Grand Rapids, MI 49503 (E-mail: timek@wmets.com).

$0022-5223 / \$ 36.00$

Copyright (c) 2014 by The American Association for Thoracic Surgery

http://dx.doi.org/10.1016/j.jtcvs.2014.02.051
}

been increasingly recognized. ${ }^{3,4}$ Annular septal-lateral (SL) reduction is paramount for the efficacy of annuloplasty rings ${ }^{5}$ and, in experimental models, has also been shown to simultaneously alter ventricular geometry. ${ }^{6}$ This increasing body of clinical and experimental data have led to the development of etiology-specific annuloplasty rings to treat IMR by addressing annular and subvalvular geometric perturbations with a customized annular prosthesis. The GeoForm annuloplasty ring (Edwards Lifesciences, Irvine, Calif) offers significant annular septal reduction while maintaining the annular orifice area and has been specifically developed to treat IMR. ${ }^{7}$ The 3-dimensional shape of the prosthesis is designed to enhance leaflet coaptation and simultaneously alter the subvalvular geometry. However, the clinical efficacy of this novel annuloplasty ring and the mid-term results have not yet been firmly established. We reviewed the clinical results of all patients with IMR who had been implanted with the GeoForm ring at our center during a 7-year period.

\section{METHODS}

The institutional review board approved our study, which was in full compliance with its policies and procedures. We performed a retrospective review of the data from all patients entered in our local Society of Thoracic 


$$
\begin{aligned}
& \text { Abbreviations and Acronyms } \\
& \text { IMR = ischemic mitral regurgitation } \\
& \text { LV }=\text { left ventricular } \\
& \text { NYHA = New York Heart Association } \\
& \text { SF-36 = Medical Outcomes Study 36-item } \\
& \text { short-form health survey } \\
& \text { SL } \quad \text { septal-lateral }
\end{aligned}
$$

Surgeons database from January 2005 to January 2011 who had undergone implantation of the GeoForm annuloplasty ring for treatment of IMR. The general indication for clinical use of the GeoForm prosthesis in our practice was patients with significant (at least moderate) functional or IMR with a low ejection fraction $(<35 \%)$ and a significantly dilated mitral annulus and left ventricular (LV) cavity. All patients were included, regardless of the preoperative degree of mitral insufficiency and additional or previous cardiac procedures. All surgeries were performed at a single center within a university-affiliated cardiac surgical practice. The operative procedure was performed according to surgeon preference but predominantly included clamping of the internal mammary artery in cases with previous patent coronary grafts, mild systemic hypothermia, and both anterograde and retrograde cold blood cardioplegia delivery. The heart was typically arrested with cold anterograde blood cardioplegia, and, subsequently, cold retrograde blood cardioplegia was redelivered every 15 to 20 minutes throughout the procedure. A septal temperature probe was used, and a temperature $<10^{\circ} \mathrm{C}$ was achieved with each dose. Before crossclamp removal, $500 \mathrm{~mL}$ of warm retrograde cardioplegia was delivered, followed by $5 \mathrm{mi}-$ nutes of warm blood retrograde perfusion as a "hot shot." The implanted rings were sized using the clinical criteria of intertrigonal distance and subsequent downsizing of 1 to 2 sizes, depending on the degree of LV dysfunction, patient size, and LV chamber remodeling. All patients received either a 26- or 28-mm ring. The ring was implanted using multiple (15-20) 2-0 Ethibond sutures to better distribute the tension and reduce the risk of prosthesis dehiscence. The anterior portion of the ring was tied down first. Subsequently, alternating sutures were tied, starting from each trigone and working toward the center of the posterior annulus. The midposterior "hump" of the ring was tied last to minimize the chance of prosthesis dehiscence from high suture tension. The perioperative mortality and 30-day events were queried directly from The Society of Thoracic Surgeons database. Follow-up regarding late mortality was conducted using the Social Security Death Index and was 100\% complete. For all deceased patients, death certificates were obtained to determine the cause of death. The EuroSCORE II score was calculated using the available on-line calculator and preoperative risk factors. The pre- and postoperative echocardiograms (294 total) were reviewed by a single experienced echocardiographer (C.T.A.) to assess the degree of MR and ventricular function and geometry preoperatively, in the early postoperative period (intraoperative or within 6 months), and at the last available follow-up visit beyond 6 months. The degree of MR was determined by visual inspection of the extent of the regurgitant jet traversing the left atrium, reversal of flow in the pulmonary veins, and measurements of the vena contracta width. MR was graded using these standard clinical criteria on a 0 to $4+$ scale. Surviving patients were interviewed by telephone to complete the Medical Outcomes Study 36-item short-form health survey (SF-36) questionnaire and valve-related morbidity questions. The SF-36 evaluates 8 dimensions of health: physical functioning, role limitations due to physical problems, bodily pain, general health perception, vitality, social function, role limitations due to emotional problems, and mental health. In addition, the SF-36 provides summary scales for overall physical and mental health. Valverelated morbidity was reported according to established guidelines. ${ }^{8}$ The preoperative patient characteristics and postoperative morbidity were defined using The Society of Thoracic Surgeons criteria. All patient data are reported as the group mean \pm standard deviation. The Kaplan-Meier method was used to determine actuarial survival.

\section{RESULTS}

A total of 86 patients underwent implantation of the GeoForm annuloplasty ring. The preoperative clinical characteristics are listed in Table 1 . Most patients had $\geq 3+$ MR, and the mean MR grade was $3.1 \pm 0.8$ (range, 1-4+). Most regurgitant jets were centrally oriented, and the mean preoperative annular SL dimension was $3.9 \pm 0.5$ $\mathrm{cm}$. The operative data and associated cardiac procedures are summarized in Table 2 . The cardiopulmonary bypass time and aortic crossclamp time were prolonged, reflecting complex operative procedures, with a $20 \%$ rate of reoperative surgery. However, most of the prolonged operative time was driven by an aggressive revascularization strategy, because $78 \%$ of patients had undergone concurrent coronary artery bypass grafting, and the mean number of grafts was $3.5 \pm 1.4$. The 30 -day mortality was $5.8 \%$ ( 5 of 86 ), and the rate of renal failure and re-exploration for bleeding was $11.1 \%$ (10 of 86 ) and $2.3 \%$ ( 2 of 86 ), respectively. No perioperative neurologic events occurred. The mean postoperative ventilator time was $54 \pm 127$ hours, and the intensive care unit stay was $125 \pm 162$ hours.

At a mean follow-up period of $50 \pm 17$ months postoperatively, 49 of the 65 surviving patients $(75 \%)$ were interviewed by telephone to assess valve-related morbidity and complete the SF-36 questionnaire. Of the 49 patients, 18 $(37 \%)$ were in New York Heart Association (NYHA) class $1,21(43 \%)$ were in NYHA class II, and $10(20 \%)$ were in either NYHA class III or IV. During the follow-up interval, $4(8 \%)$ of the 49 patients developed bleeding complications, $3(6 \%)$ had neurologic events, $1(2 \%)$ had endocarditis, $29(60 \%)$ were receiving anticoagulant therapy, and 27 $(55 \%)$ had permanent pacemakers. The high permanent pacemaker rate was reflective of an aggressive strategy using biventricular pacing for potential improvement of LV function, because $43 \%$ of the study patients had received either a biventricular pacer or a LV pacing lead at GeoForm ring implantation. No valve-related reoperations were required, although 1 patient with tricuspid valve endocarditis was denied surgery because of end-stage cirrhosis. Of the 49 patients, 16 died during the follow-up period, with 8 from cardiac causes. Survival at 1,3 , and 5 years postoperatively was $87 \%, 81 \%$, and $75 \%$, respectively (Figure 1).

All patients had undergone early echocardiography (either intraoperatively or within 6 months), revealing a mean MR grade of $0.2 \pm 0.4$ (range, $0-4+$ ) in the early postoperative period. Of the 81 patients surviving the perioperative period, $61(75 \%)$ had undergone echocardiography $>6$ months postoperatively. In these patients, at the last follow-up point (mean, $41 \pm 18$ months), the mean MR grade was $0.7 \pm 0.7$, with 2 patients developing $\geq 2+$ recurrent mitral insufficiency. The freedom from recurrent 
TABLE 1. Patient characteristics

\begin{tabular}{lc}
\hline \multicolumn{1}{c}{ Variable } & Value \\
\hline Patients (n) & 86 \\
Age (y) & $66 \pm 12$ \\
Male gender & $51(59)$ \\
EF $(\%)$ & $31 \pm 11$ \\
EuroSCORE II (\%) & 9.3 \\
MR grade (1-4+) & \\
$2+$ & $27(31)$ \\
$3+$ & $25(29)$ \\
$\quad 4+$ & $34(40)$ \\
NYHA class III-IV & $71(83)$ \\
Diabetes & $18(21)$ \\
COPD & $9(10)$ \\
Atrial fibrillation & $11(13)$ \\
Vascular disease & $12(14)$ \\
Urgent presentation & $33(38)$ \\
Previous MI & $59(68)$ \\
\hline Data presented as mean \pm standard deviation or $\mathrm{n}(\%)$, unless otherwise noted. $E F$, \\
Ejection fraction; $M R$, mitral regurgitation; $N Y H A$, New York Heart Association; \\
COPD, chronic obstructive pulmonary disease; $M I$, myocardial infarction.
\end{tabular}

$\geq 2+$ MR at 5 years was $86 \%$ (Figure 2). Paired analysis of the ejection fraction revealed a significant increase relative to the preoperative measurements $(39 \% \pm 14 \%$ vs $33 \% \pm$ $12 \% ; P=.003)$. Significant LV remodeling was also observed, with the LV end-diastolic diameter decreasing from $6.0 \pm 0.8 \mathrm{~cm}$ to $5.4 \pm 0.9 \mathrm{~cm}(P=.0002)$ and the $\mathrm{LV}$ end-systolic diameter decreasing from $5.0 \pm 09 \mathrm{~cm}$ to $4.4 \pm 1.1 \mathrm{~cm}(P=.0002)$. The peak and mean diastolic mitral valve gradient was $12.0 \pm 4.5$ and $4.6 \pm 1.9 \mathrm{~mm} \mathrm{Hg}$, respectively.

The quality of life data are summarized in Figure 3. No significant differences were found between our patient group and the general age-matched population for the individual health measures of the SF-36 or the composite mental or physical health score. When compared by individual age group $(45-54,55-64$, and $>65$ years), the

TABLE 2. Operative data

\begin{tabular}{lc}
\hline \multicolumn{1}{c}{ Variable } & Value \\
\hline Ring size & \\
$26 \mathrm{~mm}$ & $60(70)$ \\
$28 \mathrm{~mm}$ & $26(30)$ \\
CABG & $67(78)$ \\
TV procedure & $21(24)$ \\
Reoperative surgery & $17(20)$ \\
Maze & $11(13)$ \\
IABP & $10(12)$ \\
LV lead/PPM & $37(43)$ \\
CPB time (min) & $237 \pm 74$ \\
Crossclamp time (min) & $190 \pm 64$ \\
\hline
\end{tabular}

Data presented as $\mathrm{n}(\%)$ or mean \pm standard deviation. $C A B G$, Coronary artery bypass grafting; $T V$, tricuspid valve; $I A B P$, intra-aortic balloon pump; $P P M$, permanent pacemaker; $C P B$, cardiopulmonary bypass; $L V$, left ventricular.

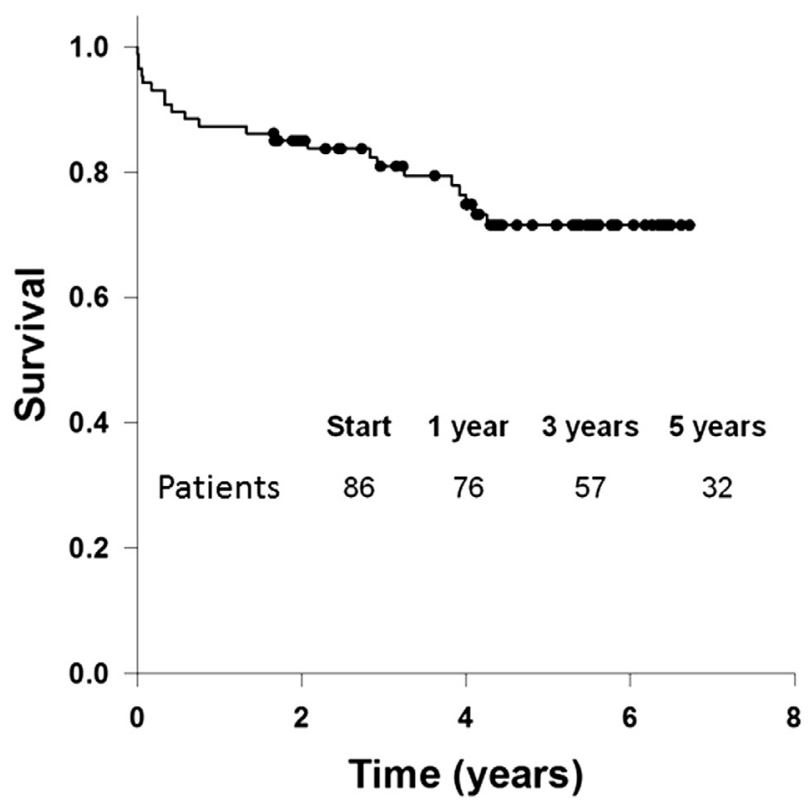

FIGURE 1. Kaplan-Meier curve summarizing actuarial patient survival.

GeoForm patients in category of the role limitations due to physical problems fared worse than the age-matched controls $(P=.03)$ in the 45 - to 54-year age group. However, significantly better scores were achieved than those for the age-matched controls in bodily pain in the 45- to 54-year age group, social function in the $>64$-year age group, mental health in the 55- to 64-year age group, and $>64$-year age group and composite score of mental health in the 55- to 64-year and $>64$-year age groups $(P<.05$ for all).

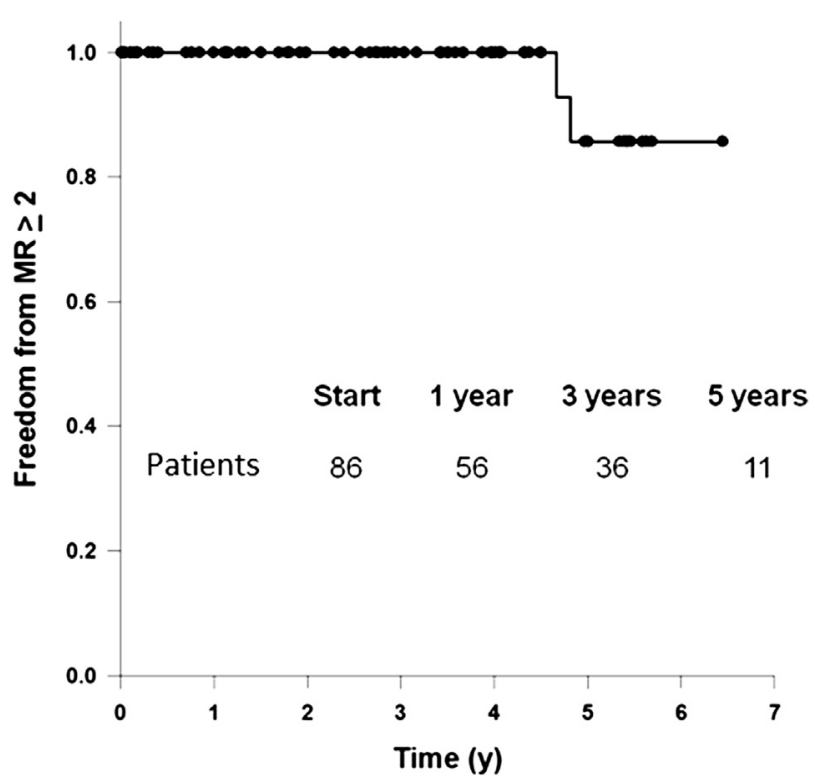

FIGURE 2. Kaplan-Meier curve representing freedom from moderate $(2+)$ or greater mitral regurgitation $(M R)$ during postoperative follow-up. 


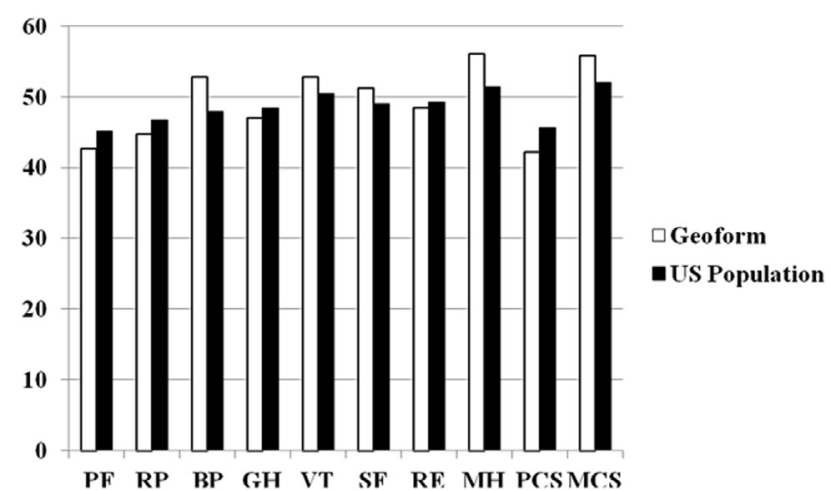

FIGURE 3. Bar graph summarizing mean Medical Outcomes Study 36-item short-form health survey sores by category for GeoForm patients (white bars) and age-matched US population norms (black bars). The 8 dimensions of health included were physical functioning $(P F)$, role limitations due to physical problems $(R P)$, bodily pain $(B P)$, general health perception $(G H)$, vitality $(V T)$, social function $(S F)$, role limitations due to emotional problems $(R E)$, and mental health $(M H)$. The composite scales for overall physical $(P C S)$ and mental $(M C S)$ health are also shown. No significant difference in the mean scores was observed for any of these categories.

\section{DISCUSSION}

IMR has continued to frustrate surgeons and cardiologists alike. The pathophysiology of IMR is complex, involving both the valvular and the subvalvular apparatus. The simple surgical "fix" of undersized ring annuloplasty has begun to illustrate its shortcomings, with significant recurrent mitral insufficiency even at short-term followup. ${ }^{9}$ The GeoForm annuloplasty ring has been specifically designed to address the annular and leaflet perturbations associated with IMR and potentially to alter the subvalvular geometry and improve the clinical results. In our clinical series, we found this prosthesis to be associated with good perioperative outcomes and distant survival $\leq 5$ years postoperatively. Implantation of the GeoForm ring in patients with IMR yielded a very low rate of recurrent mitral insufficiency and good functional status of the patients at follow-up.

Etiology-specific annuloplasty rings have been developed to customize mitral annular remodeling according to the particular pathophysiology of mitral insufficiency. Currently, the IMR ETiologix ring, GeoForm ring (both Edwards Lifesciences), and Rigid Saddle Annuloplasty Ring (St Jude Medical, St Paul, Minn) are the annuloplasty prostheses specifically available for clinical repair of IMR and offering a variable degree of SL annular reduction. ${ }^{10}$ Dilatation of this annular dimension has been thought to be central to the pathogenesis of IMR. In the present series, the GeoForm ring performed well in a high-risk IMR patient population with a low ejection fraction and high EuroSCORE II score. These outcomes have been are corroborated by a recent Italian series, ${ }^{11}$ with similar survival $(81 \%)$ and rate of recurrent moderate IMR at 3.5 years postoperatively. These investigators did not observe significant transmitral pressure gradients either at rest or during exercise, ${ }^{11}$ supporting our findings. Our study did not include stress testing; however, $80 \%$ of our patients at the last follow-up point were either in NYHA class I or II and their subjective well-being, determined from the SF-36 scores, was equal to that of the age-matched general population, making the possibility of clinical mitral stenosis less likely. Significant LV remodeling was observed in our study, with an increase in the LV ejection fraction. However, it would be difficult to ascertain whether the positive effect of surgery on ventricular geometry and performance resulted from annular remodeling or concurrent procedures, because a large proportion of patients underwent simultaneous coronary artery bypass grafting. A number of patients had also undergone placement of a LV lead or revision of a pacemaker at surgery for subsequent biventricular pacing. This treatment modality alone can reduce IMR and contribute to LV remodeling. ${ }^{12,13}$ Most likely, the combination of these factors and prevention of volume overload through durable control of mitral insufficiency contributed to the observed effects on the LV geometry.

It would be tempting to speculate that the SL annular reduction achieved with implantation of the GeoForm ring directly affected the subvalvular geometry, because isolated SL reduction of $30 \%$ has been shown to abolish chronic ovine $\mathrm{IMR}^{14}$ and reposition the posterior papillary muscle. ${ }^{15}$ Undersized suture annuloplasty has also been demonstrated to reduce the LV radius of curvature, ${ }^{6}$ demonstrating the effect of an annular intervention on the subvalvular geometry. The SL diameter of a $26-\mathrm{mm}$ and a $28-\mathrm{mm}$ GeoForm ring is $17.1 \mathrm{~mm}$ and $18.2 \mathrm{~mm},{ }^{10}$ respectively, and, as such, the mean reduction in the SL size in our patients was $>50 \%$ and would be expected to exert influence on the geometry of the subvalvular apparatus. However, in experimental acute IMR, the GeoForm ring has not been shown to offer a greater reduction in the leaflet tenting area than prostheses with a less radical SL design. ${ }^{16}$ Furthermore, such an extreme reduction in the annular SL size could be deleterious to systolic function of the myocardial segments adjacent to the mitral annulus. ${ }^{17}$ Prospective studies of patients with IMR and implanted with the GeoForm ring using serial magnetic resonance imaging to precisely evaluate the annular, subvalvular, and ventricular geometry are needed to define the direct effect of this prosthesis on LV remodeling.

The inherent shape of the GeoForm ring radically changes the annular geometry from a D-shaped native annulus to a rectangular shape. Clinical concerns have been raised regarding extreme annular reduction and increased stress that could lead to ring dehiscence. This 
was not observed in the present series; however, the followup data are not complete and long-term data are yet not available. However, the implantation technique was standardized to include a high number of sutures (15-20) and tying down of the posterior annular "hump" last. Recent acute ovine studies have shown that ring implantation will be associated with increased strain, particularly in the lateral annulus, that is smallest for the flexible annuloplasty bands. ${ }^{18}$ However, the strain associated with the GeoForm ring was similar to that observed with physiologically shaped prostheses such as the Physio annuloplasty ring and the Rigid Saddle Annuloplasty Ring (St Jude Medical). The investigators concluded that the degree of annular SL reduction cannot predict the annular strain profile of ring prosthesis. ${ }^{18}$ From these data, the likelihood of annular disruption with the GeoForm ring might not be different from that of any other rigid prosthesis. In addition, although the GeoForm ring increased the anterior mitral leaflet strain in these same experimental animal, this again did not differ from that of other rigid annuloplasty rings ${ }^{19}$ as did not altered anterior leaflet motion. ${ }^{20}$ The posterior leaflet motion, however, was more restricted with implantation of the GeoForm ring; therefore, it is feasible that the asymmetric posterior leaflet tethering observed in some patients with IMR could be exacerbated by implantation of this device. De Bonis and colleagues ${ }^{11}$ identified asymmetric leaflet tethering as a predictor of recurrent MR in their series of patients with IMR treated with the GeoForm ring.

The GeoForm annuloplasty ring is a new tool in the surgical armamentarium aimed at reliable and lasting correction of IMR; however, the reported clinical experience has been very limited. Randomized studies have shown that ring annuloplasty and concurrent coronary artery bypass grafting will be superior to coronary artery bypass grafting alone in addressing even moderate degrees of MR. ${ }^{21}$ As such, repairing IMR provides incremental clinical benefit. However, clinical controversy still exists regarding whether the repair will be superior to valve replacement. A recent multicenter randomized trial of mitral valve repair using standard undersized complete ring annuloplasty versus mitral valve repair showed equivalent outcomes at 1 year, with superior control of mitral insufficiency seen with valve replacement. ${ }^{22}$ The almost 30\% recurrent MR rate at 1 year reported in that trial was not observed in the present study. However, it is difficult to speculate whether the use of an etiology-specific ring prosthesis would alter the findings of that important trial. The findings from the present study support the use of this prosthesis in high-risk patients with IMR, offering durable control of mitral insufficiency and favorable perioperative and distant survival. Additional studies are needed to define the long-term outcomes of the GeoForm ring and identify optimal candidates for its implantation.

\section{Study Limitations}

Although our study has presented encouraging clinical results with the use of the GeoForm ring, several limitations must be recognized to interpret the data in the appropriate context. The patient population was a heterogeneous group of preoperative morbidities and operative procedures and, as such, the isolated effect of GeoForm annuloplasty on the clinical outcomes was difficult to ascertain. However, the prosthesis provided very good control of IMR, with a low rate of recurrent insufficiency at 5 years in a study population of high-risk patients with IMR and ischemic cardiomyopathy. The echocardiographic and telephone follow-up data were only $75 \%$ complete, and potential patients with recurrent MR and/or distant morbidity might not have been included in the analysis. Ours was a retrospective study, and the postoperative imaging intervals were not standardized to allow for longitudinal assessment of MR and LV geometry. The patient criteria for implantation of the GeoForm ring were not defined but left to the discretion of the surgeon and, hence, surely introduced selection bias.

\section{References}

1. Yiu SF, Enriquez-Sarano M, Tribouilloy C, Seward JB, Tajik AJ. Determinants of the degree of functional mitral regurgitation in patients with systolic left ventricular dysfunction: a quantitative clinical study. Circulation. 2000;102:1400-6.

2. Tibayan FA, Rodriguez F, Zasio MK, Bailey L, Liang D, Daughters GT, et al. Geometric distortions of the mitral valvular-ventricular complex in chronic ischemic mitral regurgitation. Circulation. 2003;108(Suppl 1):II116-21.

3. Langer F, Rodriguez F, Ortiz S, Cheng A, Nguyen TC, Zasio MK, et al. Subvalvular repair: the key to repairing ischemic mitral regurgitation? Circulation. 2005; 112:I383-9.

4. Hvass U, Joudinaud T. The papillary muscle sling for ischemic mitral regurgitation. J Thorac Cardiovasc Surg. 2010;139:418-23.

5. Timek TA, Lai DT, Tibayan FA, Liang D, Daughters GT, Dagum P, et al. Septallateral annular cinching abolishes acute ischemic mitral regurgitation. $J$ Thorac Cardiovasc Surg. 2002;123:881-8.

6. Tibayan FA, Rodriguez F, Langer F, Liang D, Daughters GT, Ingels NB Jr, et al. Undersized mitral annuloplasty alters left ventricular shape during acute ischemic mitral regurgitation. Circulation. 2004;110(Suppl 1):II98-102.

7. Votta E, Maisano F, Bolling SF, Alfieri O, Montevecchi FM, Redaelli A. The GeoForm disease-specific annuloplasty system: a finite element study. Ann Thorac Surg. 2007;84:92-101.

8. Akins CW, Miller DC, Turina MI, et al; Councils of the American Association for Thoracic Surgery, Society of Thoracic Surgeons, European Association for Cardio-Thoracic Surgeons, Ad Hoc Liaison Committee for Standardizing Definitions of Prosthetic Heart Valve M. Guidelines for reporting mortality and morbidity after cardiac valve interventions. J Thorac Cardiovasc Surg. 2008; 135:732-8.

9. McGee EC, Gillinov AM, Blackstone EH, Rajeswaran J, Cohen G, Najam F, et al. Recurrent mitral regurgitation after annuloplasty for functional ischemic mitral regurgitation. J Thorac Cardiovasc Surg. 2004;128:916-24.

10. Bothe W, Swanson JC, Ingles NB, Miller DC. How much septal-lateral mitral annular reduction do you get with new ischemic/functional mitral regurgitation annuloplasty rings? J Thorac Cardiovasc Surg. 2010;140:117-21.

11. De Bonis M, Taramasso M, Grimaldi A, Maisano F, Calabrese MC, Verzini A, et al. The GeoForm annuloplasty ring for the surgical treatment of functional mitral regurgitation in advanced dilated cardiomyopathy. Eur J Cardiothorac Surg. 2011;40:488-95.

12. Breithardt OA, Sinha AM, Schwammenthal E, Bidaoui N, Markus KU, Franke A et al. Acute effect of cardiac resynchronization therapy on functional mitral regurgitation in advanced systolic heart failure. J Am Coll Cardiol. 2003;41: 765-70.

13. St John Sutton MG, Plappert T, Hilpisch KE, Abraham WT, Haves DL, Chinchov E. Sustained reverse left ventricular structural remodeling with cardiac 
resynchronization at one year is a function of etiology: quantitative Doppler echocardiographic evidence from the Multicenter InSync Randomized Clinical Evaluation (MIRACLE). Circulation. 2006;113:266-72.

14. Tibayan FA, Rodriguez F, Langer F, Zasio MK, Bailey L, Liang D, et al. Does septal-lateral annular cinching work for ischemic mitral regurgitation? J Thorac Cardiovasc Surg. 2004;127:654-63.

15. Tibayan FA, Rodriguez F, Langer F, Zasio MK, Bailey L, Liang D, et al. Annular or subvalvular approach to chronic ischemic mitral regurgitation? J Thorac Cardiovasc Surg. 2005;129:1266-75.

16. Bothe W, Kvitting JP, Stephens EH, Swanson JC, Liang DH, Ingels NB Jr, et al. Effect of different annuloplasty ring types on mitral leaflet tenting area during acute myocardial ischemia. J Thorac Cardiovasc Surg. 2011; 141:345-53.

17. Nguyen TC, Cheng A, Tibayan FA, Liang D, Daughters GT, Ingels NB Jr, et al. Septal-lateral annular cinching perturbs basal left ventricular transmural strains. Eur J Cardiothorac Surg. 2007;31:423-9.
18. Bothe W, Rausch MK, Kvitting JP, Echtner DK, Walther M, Ingels NB Jr, et al How do annuloplasty rings affect mitral annular strains in the normal beating ovine heart? Circulation. 2012;126(Suppl 1):S231-8.

19. Bothe W, Kuhl E, Kvitting JP, Rausche MK, Göktepe S, Swanson JC, et al. Rigid, complete annuloplasty rings increase anterior leaflet strains in the normal beating ovine heart. Circulation. 2011;124(Suppl 1):S81-96.

20. Bothe W, Kvitting JP, Swanson JC, Göktepe S, Vo N, Ingels NB, et al. How do annuloplasty rings affect mitral leaflet dynamic motion. Eur J Cardiothorac Surg. 2010;38:340-9.

21. Chan KM, Punjabi PP, Flather M, Wage R, Symmonds K, Roussin I, et al. Coronary artery bypass surgery with or without mitral valve annuloplasty in moderate functional ischemic mitral regurgitation: final results of the Randomized Ischemic Mitral Evaluation (RIME) trial. Circulation. 2012;126:2502-10.

22. Acker MA, Parides MK, Perrault LP, Moskowitz AJ, Gelijns AC, Voisine P, et al. Mitral-valve repair versus replacement for severe ischemic mitral regurgitation. N Engl J Med. 2014;370:23-32. 